\title{
Usefulness of frozen elephant trunk technique for distal aortic arch aneurysms
}

\author{
Kazuo Yamanaka', Takeshi Nishina', Yuji Sekine', Syun Sato', Masashi Yada', Yuichi Tara', Atushi \\ Iwakura ${ }^{2}$, Masatoshi Fujita ${ }^{3}$ \\ 'Department of Cardiovascular Surgery, Nara Prefecture General Medical Center, Nara 630-8581, Japan. \\ ${ }^{2}$ Department of Cardiovascular Surgery, Tenri Hospital, Tenri 632-8552, Japan. \\ ${ }^{3}$ Department of Cardiovascular Medicine, Uji Hospital, Uji, Kyoto 611-0011, Japan.
}

Correspondence to: Dr. Kazuo Yamanaka, Executive Director, Cardiovascular Center, Nara Prefecture General Medical Center, 2-897-5 Shichijo-nishi, Nara 630-8581, Japan. E-mail: k-yamanaka@nara-hp.jp

How to cite this article: Yamanaka K, Nishina T, Sekine Y, Sato S, Yada M, Tara Y, Iwakura A, Fujita M. Usefulness of frozen elephant trunk technique for distal aortic arch aneurysms. Vessel Plus 2020;4:39. http://dx.doi.org/10.20517/2574-1209.2020.46

Received: 2 Sep 2020 First Decision: 19 Oct 2020 Revised: 9 Nov 2020 Accepted: 18 Nov 2020 Published: 10 Dec 2020

Academic Editor: Antonio Nenna Copy Editor: Cai-Hong Wang Production Editor: Jing Yu

\begin{abstract}
Aim: The effectual use of frozen elephant trunk (FET) has been for total aortic arch replacement (TAR) of acute aortic dissection because of positive aortic remodeling. However, the use of FET in the non-dissecting aortic arch aneurysm is still contr oversial. We aim to investigate the outcomes of TAR using the FET technique for distal aortic arch aneurysms.
\end{abstract}

Methods: Between August 2014 and February 2020, 40 patients (35 males, mean age 77.0 years) underwent TAR by using the FET technique with the J Graft Open Stent Graft for distal aortic arch aneurysms including 8 patients with shaggy aorta. In 5 of 40 patients, coronary bypass grafting was concomitantly performed. We followed up for 29.0 months.

Results: The mortality were $0 \%$. Stroke occurred in three patients (7.5\%) one of whom had shaggy aorta, paraparesis in one patient (2.5\%) who recovered fully, and respiratory complication in two patients (5.0\%). There was no recurrent nerve palsy. During the follow-up period, death had no relationship with aortic disease.

Conclusion: We conclude the FET has the potential to improve TAR for distal aortic arch aneurysms.

Keywords: Aortic arch aneurysm, frozen elephant trunk, open stent, left recurrent nerve palsy, shaggy, total aortic arch replacement

cc) (i) (c) The Author(s) 2020. Open Access This article is licensed under a Creative Commons Attribution 4.0 International License (https://creativecommons.org/licenses/by/4.0/), which permits unrestricted use, sharing, adaptation, distribution and reproduction in any medium or format, for any purpose, even commercially, as long as you give appropriate credit to the original author(s) and the source, provide a link to the Creative Commons license, and indicate if changes were made.

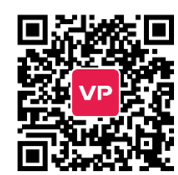




\section{INTRODUCTION}

The frozen elephant trunk (FET) technique was initially developed in 1994 as one of the first types of hybrid surgery ${ }^{[1]}$ to combine endovascular treatment and open surgery and has since been disseminated worldwide. This technique has been widely adopted as a single-stage open aortic repair technique for non- dissecting arch aneurysm with a downstream extension or a first-stage procedure ${ }^{[2]}$, followed by a second open repair or thoracic endovascular aortic repair. In cases of acute or chronic aortic dissection, this technique is a useful additional total aortic arch replacement (TAR) procedure; in such cases, FET is used to reinforce the distal anastomosis of the TAR and close the false lumen of the descending aorta ${ }^{[3]}$. In Japan, handmade FET device had been used in some hospitals since 1994. The first commercial product (JG open stent) was introduced in 2014 and modified to Frozenix in 2017. Although this graft has been widely used with concomitant TAR in Japan, the indication for non-dissecting distal aortic arch aneurysm is still controversial $^{[4]}$. We studied the outcomes of TAR by the use of the FET technique for distal aortic arch aneurysms.

\section{METHODS}

Between August 2014 and February 2020, 40 patients underwent TAR for distal aortic arch aneurysms using the FET technique at Tenri Hospital and Nara Prefecture General Medical Center. Informed consent was obtained from all patients on their information. We have followed up all patients until June 2020. The preoperative patient characteristics are listed in Table 1. The age at surgery was $77.0 \pm 6.0$ years (16 octogenarians, range 59-87 years), and the number of males was 35 (87.5\%). The number of fusiform distal aneurysm was over half. The rate of saccular type aneurysm and the extension of aneurysm was $35 \%$ and $10 \%$. Surgical indication of fusiform distal aneurysm is more than $55 \mathrm{~mm}$ of diameter. But the indication of saccular type aneurysm depends on configuration, diameter and enlargement rate. Preoperative comorbid diseases were hypertension in 32 patients $(80.0 \%)$, dyslipidemia in 16 (34.7\%), chronic obstructive pulmonary disease in $13(32.5 \%)$, coronary artery disease in $10(25.0 \%)$, stroke in $9(22.5 \%)$, diabetes mellitus in $9(22.5 \%)$ and chronic renal dysfunction, (defined serum creatinine $\geq 1.2 \mathrm{mg} / \mathrm{dL}$, in 10 patients (25\%). Six patients of them (15\%) had aortic surgery previously.

\section{FET selection}

We used Frozenix (or JG Open Stent) in all cases. The available sizes of Frozenix as follows, the diameter of Frozenix: $21,23,25,27,29,31,33,35,37,39 \mathrm{~mm}$. The length of stented graft: $6,9,12 \mathrm{~cm}$. The optimal FET was selected with use of preoperative computed tomography. The length of FET was decided by measuring the distance between the site of aortic resection and the intended location of the descending aorta. We rarely use the unstented part of FET and therefore removed all but $1 \mathrm{~cm}$ of the unstented part to prevent graft kinking. In other word, the distance from the distal end of FET should be no more than the stented part $+1 \mathrm{~cm}$. The diameter of FET was approximately $10 \%$ greater than the diameter of the descending aorta at the planned location of attachment.

\section{Operative procedure}

The operation was performed through median sternotomy. We used 2 main arterial cannulation sites; the ascending aorta in most of patients and the axillary artery in the shaggy aorta. Venous cannula was inserted into right atrium. Subsequently, the total extracorporeal circulation was initiated. After cooling to achieve a bladder temperature of $25^{\circ} \mathrm{C}$, the ascending aorta was cross-clamped, and cold blood cardioplegia was infused to the aortic root. After circulatory arrest, the proximal left subclavian artery was ligated, and we started cerebral perfusion $(200 \mathrm{~mL} / \mathrm{min}$ ) via an $8-\mathrm{mm}$ graft anastomosed to the left axially artery. After aortic arch incision, selective cerebral perfusion was established from the brachiocephalic artery $(400 \mathrm{~mL} / \mathrm{min})$ and the left common carotid artery $(200 \mathrm{~mL} / \mathrm{min})$. Total cerebral perfusion was $800 \mathrm{~mL} / \mathrm{min}$ and was performed by one roller pump ${ }^{[5]}$. We closely monitor near infrared spectroscopy and artery pressure in 
Table 1. Preoperative patient characteristics

\begin{tabular}{ll}
\hline Number & 40 \\
Age & $77.0 \pm 6.0$ years $(59-87)$ \\
Male & $35(87.5 \%)$ \\
Type of aneurysms & \\
$\quad$ Fusiform distal aneurysm & $21(52.5 \%)$ \\
$\quad$ Saccular type aneurysm & $14(35 \%)$ \\
$\quad$ The extension of aneurysm & $4(10 \%)$ \\
$\quad$ Penetrating aortic ulcer & $1(2.5)$ \\
Hypertension & $32(80.0 \%)$ \\
DM (insulin) & $9(22.5 \%)$ \\
DL & $16(34.7 \%)$ \\
CVD & $19(47.5 \%)$ \\
Renal dysfunction (Cr $\geq 1.2)$ & $10(25.0 \%)$ \\
COPD & $13(32.5 \%)$ \\
Precious aortic surgery & $6(15.0 \%)$ \\
\hline
\end{tabular}

DM: diabetes mellitus; DL: dyslipidemia; CVD: cerebrovascular disease; Cr: creatinine; COPD: chronic obstructive pulmonary disease
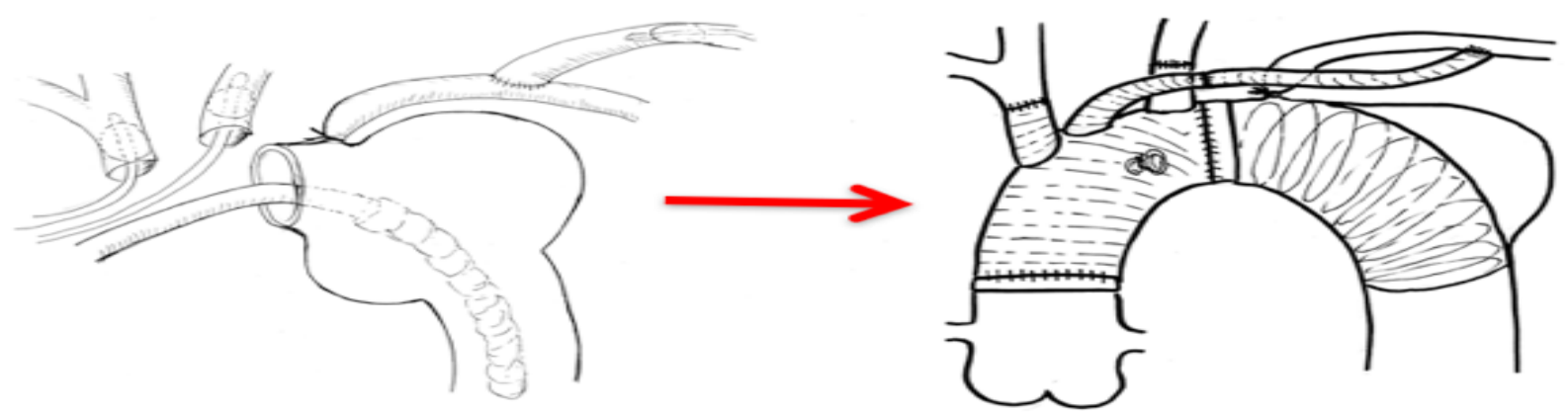

Figure 1. Procedure of total arch replacement with the frozen elephant trunk technique. The frozen elephant trunk was inserted through the transection site into the intended distal landing portion by guiding transesophageal echocardiography. We constructed the distal anastomosis with a separated 4-branched graft and antegrade systemic circulation was restarted through the side branch of the graft. The left common carotid artery and brachiocephalic artery were then anastomosed to their respective graft branches. After completion of the proximal anastomosis, the aortic graft was declamped and the $8 \mathrm{~mm}$ graft connected to the left subclavian artery was finally anastomosed with one branch of the graft

bilateral upper extremities under cerebral perfusion in all cases. The aortic arch was dissected transversely between the left common carotid artery and the left subclavian artery. The FET was inserted through the transection site into the intended distal landing portion, which was positioned up to the T8 level to prevent spinal cord injury (SCI). We confirmed the distal end of the FET by transesophageal echocardiography $(\mathrm{TEE})^{[6]}$. We constructed the distal anastomosis with a separated 4-branched graft that was reinforced with Teflon felt strips and antegrade systemic circulation was restarted through the side branch of the aortic arch graft and the patient was rewarmed by extracorporeal circulation. The left common carotid artery and brachiocephalic artery were then anastomosed to their respective graft branches. After completion of the proximal anastomosis, the aortic graft was declamped and the $8 \mathrm{~mm}$ graft connected to the left subclavian artery was finally anastomosed with one branch of the arch graft [Figures 1 and 2].

\section{Statistical analysis}

Continuous variables are expressed as the mean \pm standard deviation. The Kaplan-Meier method was used to estimate mid-term outcome. Statview for Windows, version 5.0 (SAS Institute Inc., Cary, NC), was used for the statistical analyses. Data were presented as mean $\pm \mathrm{SD}$, as appropriate. 


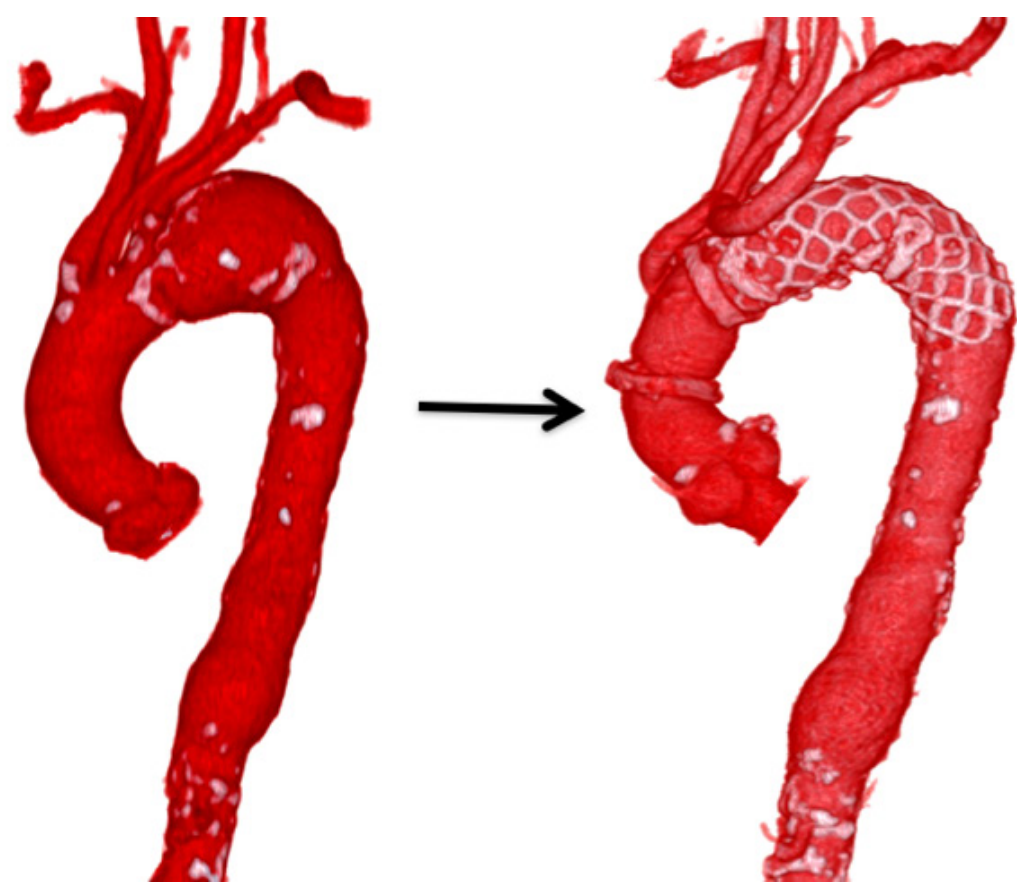

Figure 2. 3D-CT image. Left side: preoperative distal aortic arch aneurysm; right side: total arch replacement with frozen elephant trunk

Table 2. Perioperative data

\begin{tabular}{ll}
\hline Operation time & $317 \pm 66 \mathrm{~min}$ \\
CPB time & $164 \pm 28 \mathrm{~min}$ \\
SCP time & $91 \pm 24 \mathrm{~min}$ \\
CA time & $45 \pm 9 \mathrm{~min}$ \\
Cl time & $95 \pm 29 \mathrm{~min}$ \\
Bladder temperature & $25.1 \pm 0.5^{\circ} \mathrm{C}$ \\
\hline
\end{tabular}

CPB: cardiopulmonary bypass; SCP: selective cerebral perfusion; CA: circulatory arrest; $\mathrm{Cl}$ : cardiac ischemia

\section{Table 3. FET data}

\begin{tabular}{ll}
\hline FET diameter & $30.7 \pm 3.0 \mathrm{~mm}$ \\
FET length & $11.4 \pm 1.2 \mathrm{~cm}$ \\
Thoracic vertebra level of FET end & $6.4 \pm 1.0$ \\
\hline
\end{tabular}

FET: frozen elephant trunk

\section{RESULTS}

Success rate of aneurysm exclusion by the method was 100\%. There was no type II endoleak in all cases and type Ib endoleak in one case, which was not connected to the aneurysm. Perioperative data are listed in Table 2. The implantation of the FET achieved in all cases under moderate hypothermic circulatory arrest. Circulatory arrest was achieved at bladder temperature of $25.1 \pm 0.5{ }^{\circ} \mathrm{C}$. The extracorporeal circulation time was $164 \pm 28 \mathrm{~min}$, heart ischemic time and arrest time were $95 \pm 29 \mathrm{~min}$ (range 57-173 $\mathrm{min}$ ) and $45 \pm 9 \mathrm{~min}$ (range 34-73 $\mathrm{min}$ ), respectively. According to postoperative CT, the distal end of the FET was in the T5-T9 range, the mean level of T6.4 [Table 3].

Postoperative patient characteristics are listed in Table 4. Hospital mortality was $0 \%$. Mean ICU stay was $3.4 \pm 5.2$ days. Mean hospital stay was $25.3 \pm 29.6$ days. Stroke occurred in three patients $(7.5 \%)$ including one with shaggy aorta. One of the three patients developed paralysis on right side of body postoperatively, 
Table 4. Mortality and morbidity

\begin{tabular}{ll}
\hline Hospital mortality & $0(0.0 \%)$ \\
ICU stay & $3.4 \pm 5.2$ days \\
Hospital stay & $25.3 \pm 29.6$ days \\
Cerebral infarction & $3(7.5 \%)$ \\
Paraparesis & $1(2.5 \%)$ \\
Respiratory failure & $2(5.0 \%)$ \\
Recurrent nerve palsy & $0(0.0 \%)$ \\
distal embolism & $0(0.0 \%)$ \\
Re-operation for bleeding & $0(0.0 \%)$ \\
\hline
\end{tabular}

ICU: intensive care unit

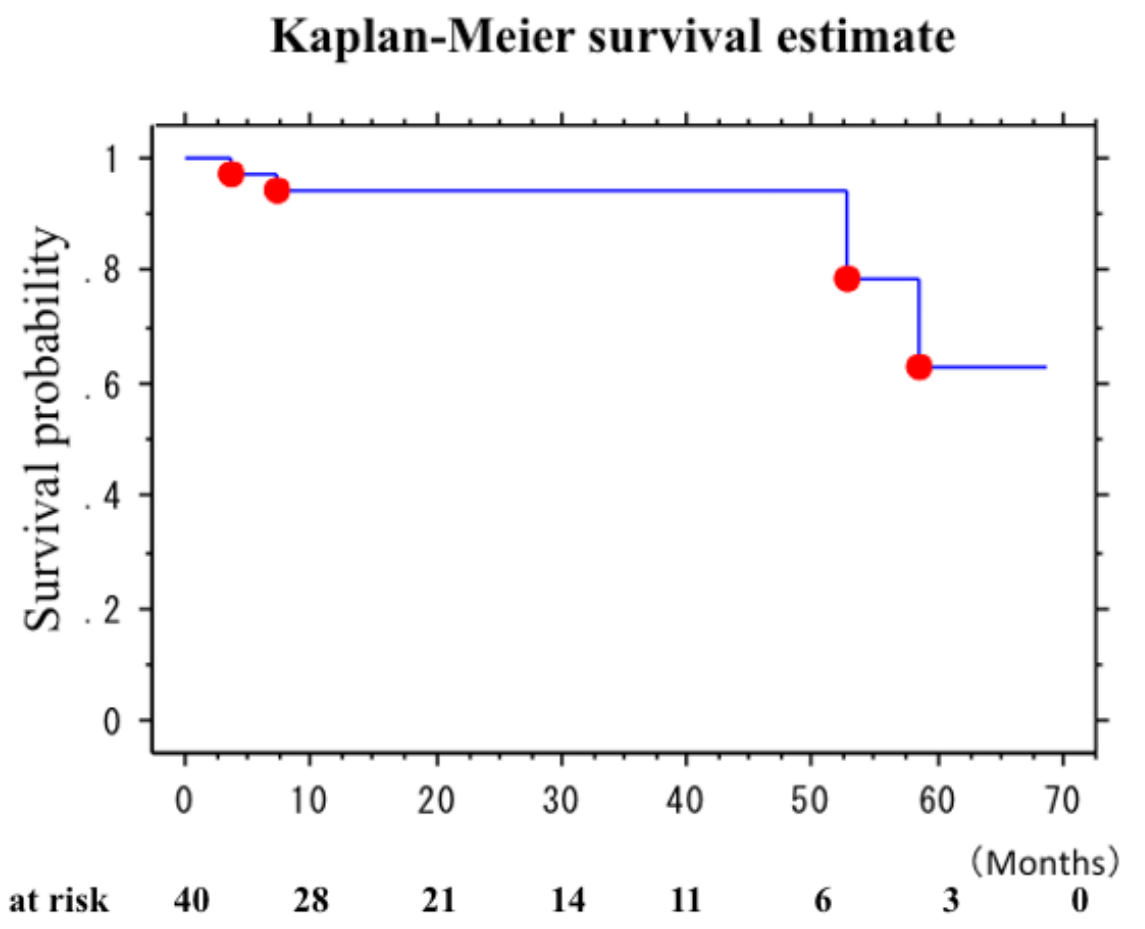

Figure 3. Kaplan-Meier survival curve for overall survival in patients with total arch replacement with frozen elephant trunk. The cause of death was not related to aortic disease. The survival rate at three years after the frozen elephant trunk was $94.3 \%$

which had disappeared before discharge. The second patient developed paralysis on left side of body while the third patient had small cerebral infarction due to right internal capsule. Paraparesis was reported in one patient $(2.5 \%)$ who recovered fully and discharged with normal gait. Respiratory complication was reported in two patients $(5.0 \%)$ who had acute respiratory distress syndrome required tracheotomy. No patients had distal embolism nor new left recurrent nerve palsy.

\section{Postoperative follow-up}

All patients were followed up for 29.0 months (range 5-68 months), during which, four patients died. The causes of death were pneumonia in two patients (postoperative 3 and 52 months) and cancer in two patients (postoperative 7 and 58 months). The cause of death had no relationship with aortic disease. The survival rate at three years after the FET was 94.3\% [Figure 3].

\section{DISCUSSION}

"Open stent" graft implantation in Japan has become established worldwide as the FET technique since the launch of commercially available products for the European and Chinese markets in the 2000s. In Japan, 
Frozenix was introduced in 2014, and up to November 2016 the accumulated clinical experience has exceed 4,000 cases. Frozenix, which consists of double-layered oval- shaped nitinol stents, maintains its shape when flexed, thus preventing undue force on the curved aortic wall ${ }^{[3]}$. A soft polyester sheath delivery system composes of a slippery material to prevent damage to the descending aorta wall during insertion. Frozenix is deployed by withdrawing the sheath after the device is correctly positioned by TEE guidance. Accordingly, these features of Frozenix enable safe and reliable device insertion. There was no FET failure nor stent fracture of FET in this study. We have had no graft failure of Frozenix (JGOS) in the pilot study since 2008 from $2010^{[3]}$. However, we have the experience of late endoleak (type $\mathrm{Ib}$ ) in the pilot study, which needed re-intervention by thoracic endovascular aortic repair. The selection of appropriate size of FET (diameter and length) is very important to prevent type Ib endoleak ${ }^{[7]}$.

The FET technique should be considered suitable for patients with extensive thoracic or thoracoabdominal aortic disease if a second class IIa procedure (open repair or thoracic endovascular aortic repair) is anticipated in downstream aortic segments, according to a European position statement ${ }^{[8]}$. This way, the operative indications of the FET for non-dissecting aortic aneurysms are still limited because TAR is gold standard for the aortic arch aneurysm and the FET has the risk of SCI. The exact mechanism of SCI in the FET is unknown. Thromboembolism, long stent graft, and circulatory arrest time are supposed as factors of $\mathrm{SCI}^{[9]}$. Presumably, the most important risk factor is the distal FET position below the level of T8. We recommend using TEE, fluoroscope, or other modalities to deploy the FET more precisely ${ }^{[10,11]}$. We always use TEE for the FET technique. Our three-step method by TEE guidance is easy and safe ${ }^{[6]}$. In our institutions, there was no permanent SCI in 113 patients with the FET (48 acute aortic dissections, 19 chronic aortic dissections, and 46 non-dissecting thoracic aneurysms. Although I trust CSF drainage, we don't preoperatively place CSF drain in elective cases because SCI rate was low in our institutions and there is 5\%-7\% complication related CSF drainage. In the patient with paraparesis, we performed CSF drainage postoperatively, kept more than $80 \mathrm{mmHg}$ mean system pressure and administered Naloxone and steroid. Thereafter, paraparesis disappeared and the patient discharged by foot. The maintenance of high systemic pressure in postoperative period is another important point to prevent SCI. Our target mean systemic pressure is more than $80 \mathrm{mmHg}$. Therefore, complete hemostasis is essential. In J-ORCHESTRA study (Japanese multicenter study) ${ }^{[12]}$, the rate of SCI in the FET group was $1.6 \%$. Although the rate is still relatively higher, Japanese surgeons mostly overcame SCI due to the FET.

Another problem is distal embolism. No patient in this study had a distal embolism. Although one of 8 patients with shaggy aorta had small cerebral infarction despite isolation technique, other patients had no cerebral infarction and no distal embolism. It is speculated that insertion of the FET under circulatory arrest never induce disturbed flow or dissipation of plaque and cover the shaggy aortic wall. According to severity or location of shaggy aorta, the use of the FET may prevent distal embolism although the use of the FET in severe shaggy aorta is presumably high risk for embolism. Some surgeons have used distal perfusion from the femoral artery after FET deployment and fixation to remove debris/air and protect distal function and Some surgeons have used thoracic perfusion with balloon-tipped Foley catheter into the end of FET to reduce visceral ischemia time. Although we also tried distal perfusion by these techniques in initial some cases, we gave up distal perfusion because it is difficult to keep bloodless field, and it makes operative procedure more complicated and time-consuming.

As stated above, the complication of the FET technique has been declining in Japan and we suppose the indication of FET can extend some high-risk patients of non-dissecting thoracic aortic aneurysms as the following patients: (1) those with distal aortic arch aneurysms that cannot be treated by thoracic endovascular aortic repair involving the left subclavian artery to the ascending aorta; (2) those in whom a median approach extending to the upper middle descending aorta for distal anastomosis with pulmonary complication is difficult; (3) those requiring redo operation; and (4) and those with an advanced age or frailty who prefer to avoid left thoracotomy. 
Additionally, the FET has some attractive advantages as follows: (1) the proximalization of distal anastomosis to zone 2; (2) the simplification of technical complexity; (3) the easy deployment of the FET; (4) the shortening of lower body ischemic time; and (5) fixation of stented graft to avoid graft migration. Furthermore, postoperative hoarseness can be avoided by the use of the FET because the distal anastomosis site lies off the left recurrent nerve at the distal aortic arch. Hoarseness may induce to aspiration pneumonia in elderly patients. The left recurrent nerve palsy was $0 \%$ in TAR with the FET (40 patients) and 5\% in that without the FET (121 patients) in our other study ${ }^{[13,14]}$. Therefore, the FET technique may better preserve postoperative laryngeal function in elderly frail patients.

In conclusion, the FET attained the proximalization of distal anastomosis to zone 2 rather than zone $3^{[15]}$. It caused decreases in incidences of cardiac arrest, selective cerebral perfusion, and visceral ischemia. It further helped to reduce the incidence of recurrent nerve palsy, the ease of anastomosis, and control of hemorrhage. The FET has the potential to improve TAR for distal aortic arch aneurysms.

\section{DECLARATIONS}

\section{Authors' contributions}

Authors made substantial contributions to the conception and design of the study, and participated in drafting the article: Yamanaka K

Authors provided administrative, technical, and material support: Iwakura A

The author participated in critical revision for important conceptual and intellectual content and gave final approval of the version to be submitted to the Journal: Fujita M or more authors: Nishina T, Sekine Y

Performed data acquisition, as well as provided administrative, technical, and material support: Sato S, Yada M, Tara Y

\section{Availability of data and materials}

Not applicable.

\section{Financial support and sponsorship}

None.

\section{Conflicts of interest}

All authors declared that there are no conflicts of interest.

\section{Ethical approval and consent to participate}

Not applicable.

\section{Consent for publication}

Not applicable.

\section{Copyright}

(c) The Author(s) 2020.

\section{REFERENCES}

1. Kato M, Ohnishi K, Kaneko M, et al. New graft-implanting method for thoracic aortic aneurysm or dissection with a stented graft. Circulation 1996;94:II188-93.

2. Mestres CA, Tsagakis K, Pacini D, et al. One-stage repair in complex multisegmental thoracic aneurysmal disease: results of a multicenter study. Eur J Cardiothorac Surg 2013;44:e325-31.

3. Uchida N, Katayama A, Tamura K, Sutoh M, Kuraoka M, Ishihara H. Frozen elephant trunk technique and partial remodeling for acute type A aortic dissection. Eur J Cardiothorac Surg 2011;40:1066-71.

4. Uchida N, Katayama A, Higashiue S, et al. A new device as an open stent graft for extended aortic repair: a multicentre early experience 
in Japan. Eur J Cardiothorac Surg 2016;49:1270-8.

5. Shimizu H, Matayoshi T, Morita M, Ueda T, Yozu R. Total arch replacement under flow monitoring during selective cerebral perfusion using a single pump. Ann Thorac Surg 2013;95:29-34.

6. Yamanaka K, Ishii H. Three-step method for transesophageal echocardiography-guided implantation of the frozen elephant trunk: how to prevent spinal cord injury. Gen Thorac Cardiovasc Surg 2019;67:340-3.

7. Kandola S, Abdulsalam A, Field M, Fisher RK. Frozen elephant trunk repair of aortic aneurysms: how to reduce the incidence of endoleak and reintervention. JTCVS Techniques 2020;1-8.

8. Shrestha M, Bachet J, Bavaria J, et al. Current status and recommendations for use of the frozen elephant trunk technique: a position paper by the Vascular Domain of EACTS. Eur J Cardiothorac Surg 2015;47:759-69.

9. Thron AK. Vascular anatomy of the spinal cord: neuroradiological investigations and clinical syndromes. New York: Springer; 1988. pp. $3-65$.

10. Roselli EE, Soltesz EG, Mastracci T, Svensson LG, Lytle BW. Antegrade delivery of stent grafts to treat complex thoracic aortic disease. Ann Thorac Surg 2010;90:539-46.

11. Ikeda A, Konishi T, Matsuzaki K, Jikuya T. Radiopaque ruler-guided Frozen elephant trunk technique. Ann Vasc Dis 2016;9:352-5.

12. Okita Y. Frozen elephant trunk with Frozenix prosthesis. Ann Cardiothorac Surg 2020;9:152-63.

13. Yamanaka K. Editorial comment regarding "Total aortic arch replacement using the frozen elephant trunk technique with J Graft Open Stent Graft for distal aortic arch aneurysm”. Gen Thorac Cardiovasc Surg 2018;66:501-3.

14. Yamanaka K. Chapter of Frozen Elephant Trunk, Volume 4; Aortic surgery. Excellence in Cardioaortic Surgery. Tokyo: Nakayama shoten Ltd. In press.

15. Jakob H, Dohle DS, Piotrowski J, et al. Six-year experience with a hybrid stent graft prosthesis for extensive thoracic aortic disease: an interim balance. Eur J Cardiothorac Surg 2012;42:1018-25. 